\title{
INFLUENCIA DA DISPOSIÇÃO DAS FILEIRAS E ESPACAMENTO ENTRE PLANTAS NO RENDIMENTO DE ALHO
}

\author{
THE INFLUENCE OF ROWS AND SPASCING DISPOSITION \\ OF PLANTS ON GARLIC YIELD
}

\author{
Danton Camacho Garcia* Valmor Barnit* Luiz Augusto Dettmann ${ }^{\star \star}$
}

RESUMO

Com o objetivo de avaliar os efeitos de diferentes espaçamentos e disposição das fileiras em canteiros de alho (Allium sativum L.), cultivar Quitéria, conduziu-se um experimento no município de Rio Grande-RS. O plantio foi realizado, em 23 de julho e a colheita dos bulbos em 15 de Dezembro de 1987. O delineamento experimental utilizado foi $o$ de blocos ao acaso, com quatro repetições. Os tratamentos foram constituídos de diferentes disposição das plantas nas parcelas, variando - espaçamento $\theta$ a disposição das fileiras em relação a maior distância da parcela, cujas dimençōes foram de 4 $x 1,20 \mathrm{~m}$. As maiores produções comerciais de bulbos foram obtidas nos tratamentos: espaçamento de $25 \times$ $8 \mathrm{~cm}$ e fileiras longitudinais $(6,76 \mathrm{t} / \mathrm{ha})$; sistema de fileiras duplas espaçadas de $50 \mathrm{~cm}(6,51 \mathrm{t} / \mathrm{ha})$ e espaçamento de $25 \times 8 \mathrm{~cm}$, fileiras transversais $(6,13 \mathrm{t} / \mathrm{ha})$

Palavras-chave: alho, espaçamento, fileiras.

\section{SUMMARY}

The effects of spascing and row disposition on garlic (Allim sativum L.) yield were evaluated. The experiment was carried out in the country of Rio Grande, RS and cultivar "Quiteria" was used. The planting was realized on July 23 , and the bulbs were yielded on December 15, 1987. The experimental design used was the randomized block with, four replicates. The treatments were the diferent plant disposition in the parcels. There was variation on spacing and row disposition in relation to the greater distance of the parcels, that had dimensions of $4 \times 1,20 \mathrm{~m}$. The better comercial yields of garlic bulbs were obtained with the following tratments: Spacing of $25 \times 8 \mathrm{~cm}$ with longitudinal rows $(6.76 \mathrm{t} / \mathrm{ha})$; double dual row system, spaced $50 \mathrm{~cm}(6: 51 \mathrm{t} / \mathrm{ha})$ and spacing of $25 \times 8 \mathrm{~cm}$ with transversal rows $(6.13 \mathrm{t} / \mathrm{ha})$.

Key words: garlic, spascing, rows

\section{INTRODUÇÃO}

A produtividade da cultura do alho, depende, entre outros fatores, do peso e qualidade dos bulbilhos plantados, da adubação, dos tratos culturais e, principalmente, do número de plantas por hectare. Em geral, utilizando-se espaçamentos maiores obtém-se maior tamanho de bulbo e menor produção comercial por área e, segundo OHM \& SRIVASTAWA (1977) acréscimos na altura de plantas, diâmetro do pseudocaule, número de folhas por planta e diâmetro do bulbo são vantagens que se consegue empregando espaçamentos maiores. Ao contrário, a utilização de espaçamentos reduzidos propicia, normalmente, menor tamanho de bulbos e maior produção por área (MASCARENHAS, 1978; MUELLER \& BIASI, 1982). Tendo por objetivo a obtenção de alho-semente, SOSA \& LEANDRO (1986) observaram que, reduzindo o espaçamento, aumentou a produção de alho-semente porém com sensivel redução do peso médio de bulbilhos.

A maioria das referências sobre espaçamento para a cultura do alho indicam, como usuais, os limites de 20 a $40 \mathrm{~cm}$ entre linhas por 7,5 a $15 \mathrm{~cm}$ entre plantas na linha (COUTO, 1958; SATURNINO et al, 1976; MUELLER \& BIASI, 1982; GARCIA et al, 1984; GASTAUD \& CARINGI, 1985)

Em Minas Gerais, os produtores utilizam canteiros de $80 \mathrm{~cm}$ de largura e plantam três fileiras de plantas por canteiro. Este sistema de plantio implica nae perda de $30 \%$ da área, ocupada por sulcos de irrigação, ficando a população final em torno de 300.000 plantas por hectare (SATURNINO et al, 1976).

A população de plantas influencia diversas características das plantas de alho.

\footnotetext{
* Engenheiro Agrônomo, Professor Assistente, Departamento de Fitotecnia do Centro de Ciências Rurais (CCR) da Universidade Federal de Santa Maria (UFSM). 97119-900 - Santa Maria-RS.

** Engenheiro Agrônomo, Pesquisador do IPAGRO Secretaria da Agricultura e Abastecimento-RS, Caixa Postal 126, 96500-970 - Rio Grande, RS.
} 
No Paraná, LOPES \& BARBEDO (1990), avaliando diversos espaçamentos observaram, para as variáveis peso total de planta, diâmetro e peso médio de bulbos curados, aumentos significativos com maior espaçamento entre linhas e com menor número de plantas dentro da linha. Para peso total de plantas, o rendimento máximo foi atingido com espaçamento de $40 \mathrm{~cm}$ entre linhas e 12,5 a $15 \mathrm{~cm}$ entre plantas dentro da linha.

REGHIN (1989), avaliando espaçamentos entre plantas, dentro de fileiras espaçadas de $25 \mathrm{~cm}$, observou maior altura quando elas foram distribuídas no maior espaçamento dentro da fileira. Por outro lado não verificou influência no percentual de pseudoperfilhamento. Bulbos maiores foram obtidos nos espaçamentos maiores $(11$ e $13 \mathrm{~cm})$, e não ocorreram diferenças significativas no peso dos bulbos produzidos nos menores espaçamentos.

Em Santa Catarina, na maioria das lavouras, utiliza-se canteiros com fileiras longitudinais em número de 4 a 5 , no espaçamento de $25 \times 8 \mathrm{~cm}$; este sistema proporciona populações de 250.000 a 370.000 plantas por hectare, dependendo da área perdida entre canteiros. Encontra-se, também, lavouras com fileiras únicas, transversais aos canteiros, com espaçamento de $25 \times$ $8 \mathrm{~cm}$ (LUCINI \& CHONAN, 1986).

$\mathrm{Na}$ região Sul do Estado do Rio Grande do Sul, é comum a sistematização do terreno em canteiros. Estes têm dimenções de aproximadamente $1,20 \mathrm{~m}$ de largura entre os centros dos sulcos de drenagem e utilizase 3 a 4 fileiras de plantas sobre a largura útil de cada canteiro, obtendo-se populações de 273.000 a 363.000 plantas por hectare (GARCIA, 1984).

Como se observa, a escolha do espaçamento depende, em cada região de cultivo, de diversos fatores.

O objetivo deste trabalho foi avaliar diferentes modos de distribuição das fileiras de plantas de alho em diferentes espaçamentos, visando obter maiores rendimentos da cultura para as condições da região Sul do Estado do Rio Grande do Sul.

\section{MATERIAL E MÉTODOS}

O experimento foi realizado na Estação Experimental de Rio Grande, do IPAGRO-Secretaria da Agricultura e Abastecimento, RS, no município de Rio Grande, RS, durante $O$ ano agrícola 1987/88.

O solo é um Podzólico Vermelho Amarelo textura média e, antes da instalação do experimento, a análise do solo ( 0 a $20 \mathrm{~cm}$ de profundidade) revelou $\mathrm{pH}$ $\mathrm{H}_{2} \mathrm{O}=5,1 ; \mathrm{pH}$ SMP $=6,2 ; \mathrm{M} . \mathrm{O}=2,0 \% ; \mathrm{Al}=0,2 \mathrm{meq} / 100$ $\mathrm{ml} ; \mathrm{Ca}+\mathrm{Mg}=1,5 \mathrm{meq} / 100 \mathrm{ml} ; \mathrm{P}=13,0 \mathrm{ppm} ; \mathrm{K}=$ 32ppm; $\mathrm{Zn}=0,4 \mathrm{ppm} ; \mathrm{Cu}=0,5 \mathrm{ppm}$. As análises foram realizadas no Centro Agronômico de Pesquisas da FERTISUL S.A., em Rio Grande, RS.
A adubação foi feita a lanço, antes do plantio dos bulbilhos, nas doses de $180 \mathrm{~kg} / \mathrm{ha}$ de $\mathrm{P}_{2} \mathrm{O}_{5}$ (superfosfato triplo), $170 \mathrm{~kg} / \mathrm{ha}$ de $\mathrm{K}_{2} \mathrm{O}$ (cloreto de potássio) e $80 \mathrm{Kg} / \mathrm{ha}$ de $N$ (uréia), sendo $40 \mathrm{~kg} / \mathrm{ha}$ aplicados no plantio e o restante 30 dias depois.

O plantio dos bulbilhos foi realizado em 23 de julho de 1987, pós processo de seleção em peneiras com diferentes malhas, sendo usados aqueles retidos na peneira $2(10 \times 20 \mathrm{~mm})$, com peso médio, de $3,03 \mathrm{~g}$.

Foi utilizada, como alho-semente, a cultivar Quitéria, alho nobre de ótima qualidade e expressiva utilização nas regiōes Sul do Rio Grande do Sul e Planalto de Santa Catarina.

Os tratos culturais como, controle de plantas daninhas, pragas e doenças, irrigaçōes e outros, seguiram o sistema de produção normalmente utilizado para cultivo do alho no Rio Grande do Sul.

Os tratamentos foram constituídos de diferentes disposições das plantas nas parcelas $\theta$ indicados na Tabela 1.

0 delineamento experimental foi blocos ao acaso, com quatro repetições, e a unidade experimental tinha as dimenções de $4 \times 1,20 \mathrm{~m}$. Após a análise de variância as médias dos tratamentos foram comparadas pelo teste de Duncan ao nível de $5 \%$ de probabilidade.

Os contrastes, envolvendo grupos com mais de duas médias, foram comparados pelo teste " $t$ " ao nível de $5 \%$ de probabilidade e descritos a seguir:

Efeito do sistema tradicional de cultivo na região=

TABELA 1 - Tratamentos, espaçamento, disposição das fileiras e uso de plantas intercaladas.

\begin{tabular}{|c|c|c|}
\hline Tratamentos & $\begin{array}{l}\text { Espaçamento } \\
\text { (cm) }\end{array}$ & $\begin{array}{l}\text { Disposição das } \\
\text { Fileiras }\end{array}$ \\
\hline
\end{tabular}

$\begin{array}{llll}\mathrm{T}_{1} & 30 \times 10 & \text { Longitudinal } & - \\ \mathrm{T}_{2} & 25 \times 10 & \text { Longitudinal } & - \\ \mathrm{T}_{3} & 25 \times 08 & \text { Longitudinal } & - \\ \mathrm{T}_{4} & 20 \times 10 & \text { Longitudinal } & - \\ \mathrm{T}_{5} & 25 \times 08 & \text { Transversal } & \text { Não } \\ \mathrm{T}_{6} & 25 \times 08 & \text { Transversal } & \text { Sim } \\ \mathrm{T}_{7} & 25 \times 10 & \text { Transversal } & \text { Sim } \\ \mathrm{T}_{8} & 20 \times 10 & \text { Transversal } & \text { Não }\end{array}$

$T_{9}$ Três fileiras duplas espaçadas de $50 \mathrm{~cm}$, espaçamento de $25 \times 8 \mathrm{~cm}$; dispostas no sentido longitudinal.

\footnotetext{
1 = Uso de plantas intercaladas entre as plantas das extremidades de cada fileira.
} 
$Y_{1}=\left[T_{1}\right.$ versus $\left.\left(T_{2}, T_{3^{\prime}}, T_{4}, T_{5}, T_{6}, T_{7}, T_{8^{\prime}}, T_{9}\right)\right]$;

Efeito do sistema de fileiras duplas $=$

$Y_{2}=\left[T_{9}\right.$ versus $\left.\left(T_{2}, T_{3}, T_{4}, T_{5}, T_{6}, T_{7}, T_{8}\right)\right]$,

Efeito do sistema de plantio em fileiras longitu-

dinais $=$

$Y_{3}=\left(T_{2}, T_{3}, T_{4}\right)$ versus $\left(T_{5}, T_{6}, T_{7}, T_{8}\right)$

Efeito do uso de plantas intercaladas entre as plantas extremas, de cada fileira $=$

$Y_{4}=\left(T_{5}, T_{6}\right)$ versus $\left(T_{7}, T_{8}\right)$,

Antes da colheita foi avaliado o percentual de plantas pseudoperfilhadas e, para análise, os dados foram transformados em $\sqrt{\mathbf{x}}$.

A colheita foi realizada em 15 de dezembro de 1987 quando, aproximadamente, dois terços da parte aérea das plantas apresentavam-se amarelecidos.

Após cura a campo, por quatro dias, os bulbos foram armazenanados em varais num galpão durante 60 dias. Posteriormente fez-se a limpeza que consistiu na retirada das folhas, parte do pseudocaule, raízes e escamas externas dos bulbos. A seguir determinou-se, o número e peso dos bulbos com características comerciais e os dados foram convertidos para toneladas por hectare.

Como bulbos comerciais foram considerados aqueles que não apresentavam pseudoperfilhamento, bulbilhos expostos, chochos e com diâmetro menor que a classe comercial $1(<25 \mathrm{~mm})$.

\section{RESULTADOS E DISCUSSÃO}

De acordo com a Tabela 2, observa-se que, a medida que diminuiu o espaçamento, ocorreu aumento no rendimento e redução no peso médio dos bulbos, concordando com resultados obtidos por outros autores (MASCARENHAS, 1978; SOSA \& LEANDRO, 1986).

Os tratamentos com área útil por planta maior que $200 \mathrm{~cm}^{2}\left(T_{1}, T_{2}\right.$ e $\left.T_{7}\right)$, independente da disposição das fileiras, foram os que apresentaram menor rendimento e os resultados concordam, em parte, com os obtidos por Mueller, apud LUCINI \& CHONAN (1986) que, para bulbilhos com peso médio de 2,0 a $3,0 \mathrm{~g}$, deve-se utilizar um espaçamento com área útil por planta entre 200 a $250 \mathrm{~cm}^{2}$.

Por outro lado, tratamentos com área útil por planta de $200 \mathrm{~cm}^{2}\left(T_{3}, T_{4}, T_{5}, T_{6}, T_{8}\right)$, também independente da disposição das fileiras, foram os que apresentaram os melhores rendimentos e concordam com resultados obtidos, no mesmo local e em anos anteriores, por GASTAUD \& CARINGI (1985).

O sistema de fileiras duplas, apesar de apresentar menor peso médio de bulbos, permite a obtenção de maiores rendimentos. Uma melhor qualidade dos bulbos pode ser obtida pelo manejo de outros fatores, como o uso de alho-semente livre de vírus e mo-
TABELA 2 - Rendimento, peso médio e pseudoperfilhamento de bulbos de alho, cv. Quitéria.

$\begin{array}{cccc}\text { Tratamentos } & \begin{array}{c}\text { Rendimento } \\ (\mathrm{t} / \mathrm{ha})\end{array} & \begin{array}{c}\text { Peso médio } \\ \text { de bulbos }\end{array} & \begin{array}{c}\text { Plantas } \\ \text { Pseudoperfilhadas }\end{array} \\ & (\mathrm{g}) & (\%)\end{array}$

\begin{tabular}{llllll}
$\mathrm{T}_{1}$ & $4,6 \mathrm{~b}$ & $15,9 \mathrm{a}$ & 3,9 & $\mathrm{~d}$ \\
$\mathrm{~T}_{2}$ & $4,7 \mathrm{~b}$ & $12,3 \mathrm{bc}$ & 2,6 & $\mathrm{c}$ \\
$\mathrm{T}_{3}$ & $6,7 \mathrm{a}$ & $13,6 \mathrm{ab}$ & 3,0 & $\mathrm{c}$ \\
$\mathrm{T}_{4}$ & $5,3 \mathrm{ab}$ & $11,2 \mathrm{bc}$ & $1,5 \mathrm{bc}$ \\
$\mathrm{T}_{5}$ & $6,1 \mathrm{ab}$ & $11,4 \mathrm{ab}$ & 2,9 & $\mathrm{c}$ \\
$\mathrm{T}_{6}$ & $5,2 \mathrm{ab}$ & $13,8 \mathrm{bc}$ & $3,0 \mathrm{c}$ \\
$\mathrm{T}_{7}$ & $4,8 \mathrm{~b}$ & $11,5 \mathrm{bc}$ & $2,0 \mathrm{~b}$ \\
$\mathrm{~T}_{8}$ & $5,8 \mathrm{ab}$ & $10,7 \mathrm{bc}$ & $1,3 \mathrm{a}$ \\
$\mathrm{T}_{9}$ & $6,5 \mathrm{ab}$ & $10,2 \mathrm{c}$ & $1,2 \mathrm{a}$ \\
\hline
\end{tabular}
$\mathrm{CV}(\%)$
15,2
13,1
11,8

Médias seguidas por letras distintas diferem entre si, ao nível de $5 \%$ de probabilidade, pelo teste de Duncan.

dificaçōes nos níveis de adubação (BARNI, 1992).

A introdução do sistema de linhas duplas $\left(T_{0}\right)$ não mostrou diferença significativa em relação a média dos outros tratamentos (contraste $Y_{2}$ ). Entretanto, parece ser um sistema promissor, pois apresentou um dos maiores rendimentos e dos mais reduzidos percentuais de pseudoperfilhamento e vem sendo utilizada em lavouras comerciais de outras regiōes com relativo sucesso (LUCINI \& CHONAN, 1986).

Este sistema, pelo aumento da população de plantas poderá se adaptar as condiçōes edafoclimáticas da região de Rio Grande, RS que, segundo GARCIA (1984), exige a utilização de canteiros que provocam diminuição expressiva na área real de cultivo.

O contraste $\left(Y_{1}\right)$ entre o sistema de plantio mais utilizado na região, correspondente ao tratamento $T_{1}$, e a média dos demais não foi significativo. Entretanto, quando se examinam as médias de cada tratamento (Tabela 2), individualmente, estas sugerem a necessidade de uma redução no espaçamento entre plantas para se obter maior rendimento de bulbos.

Em média, a diferença entre os sistemas de disposição de fileiras no sentido transversal ou longitudinal da parcela não foi significativa como se pode observar pelas médias do contraste $Y_{3}$ na Tabela 3. Este resultado indica que o sentido de distribuição das fileiras nos canteiros não influenciou o comportamento das variáveis e, possivelmente, a escolha dependa de outros 
TABELA 3 - Contrastes, médias e significância do teste "t" para rendimento, peso medio de bulbos e percentual de plantas pseudoperfilhadas de alho.

CONTRASTE*

Médias

\begin{tabular}{|c|c|c|c|c|c|c|}
\hline & \multicolumn{2}{|c|}{$\begin{array}{l}\text { Rendimento } \\
\text { (t/ha) }\end{array}$} & \multicolumn{2}{|c|}{$\begin{array}{l}\text { Peso médio de } \\
\text { bulbos }(\mathrm{g})\end{array}$} & \multicolumn{2}{|c|}{$\begin{array}{l}\% \text { de plantas } \\
\text { Pseudoperfilhadas }\end{array}$} \\
\hline \multirow[t]{2}{*}{$Y_{1}$} & 4.6 versus. & 5.6 & 15.9 versus. & 11.8 & 3.9 versus. & 2.2 \\
\hline & A & A & A & B & B & A \\
\hline \multirow[t]{2}{*}{$Y_{2}$} & 6.5 versus. & 5.5 & 10.2 versus. & 12.1 & 1.2 versus. & 2.5 \\
\hline & A & A & A & $A$ & A & B \\
\hline \multirow[t]{2}{*}{$Y_{3}$} & 5.6 versus. & 5.5 & 12.4 versus. & 11.8 & 2.4 versus & 2.3 \\
\hline & A & A & A & A & A & A \\
\hline \multirow{2}{*}{$Y_{4}$} & 5.6 versus. & 5.3 & 12.6 versus. & 11.1 & 2.9 versus. & 1.7 \\
\hline & A & A & A & A & B & A \\
\hline
\end{tabular}

Médias seguidas por mesma letra, na horizontal, não diferem entre si pelo teste de " $\mathrm{t}$ " ao nível de $5 \%$ de probabilidade.

$$
\begin{aligned}
& { }^{*} Y_{1}=\left[T_{1} \text { versus }\left(T_{2}, T_{3}, T_{4}, T_{5}, T_{6}, T_{7}, T_{8}, T_{9}\right]\right. \text {; }
\end{aligned}
$$

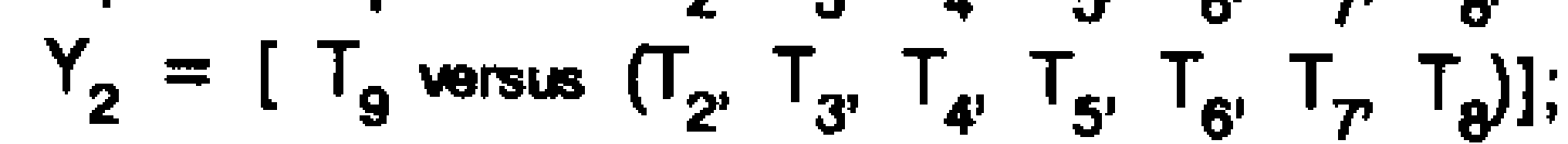

$$
\begin{aligned}
& Y_{3}=\left(T_{2}, T_{3}, T_{4} \text { versus }\left(T_{5}, T_{6}, T_{7}, T_{8}\right)\right. \text {; } \\
& Y_{4}=\left(T_{5}, T_{6}\right) \text { vorsus }\left(T_{7}, T_{8}\right) \text {. }
\end{aligned}
$$

fatores tais como utensílios para plantio, mão-de-obra disponivel e métodos de controle de plantas daninhas.

No sistema de plantio com fileiras longitudinais e com distância fixa de $10 \mathrm{~cm}$ entre plantas, fez-se análise de regressão polinomial ajustando uma equação para a rendimento de bulbos em função dos níveis 20, 25 e $30 \mathrm{~cm}$ do fator distância entre as fileiras. A equação ajustada $\hat{Y}=7,87833+0,0127 X, R^{2}=0,99$, indica que 0 rendimento de bulbos decresce proporcionalmente com - aumento da distância entre fileiras e concorda com resultados obtidos por MUELLER \& BIASI (1982).

Considerando o sistema de linhas transversais, não se verificou diferenças significativas (contraste $Y_{4}$ ) para rendimento e peso médio de bulbos, introduzindo plantas intercaladas entre as linhas nas extremidades. Entretanto, os resultados indicaram uma redução significativa no percentual de plantas pseudoperfilhadas (Tabela 2). Sugere-se o uso dessas plantas pelo aumento que proporcionam na população final e pela redução que podem provocar nos índices de pseudoperfilhamento, o que concorda com resultados semelhantes obtidos por outros autores (MUELLER \& BIASI, 1990).

Conclui-se que é possível obter aumento de produtividade, com alteraçōes nos níveis do fator espaçamento entre plantas. A escolha deste, entretanto, também deve ser definida em função, da quantidade e custo do alho-semente, da finalidade da produção (se ob- tenção de alho-semente ou para consumo in natura) $e$ das metas de produtividade a serem alcançadas.

\section{REFERÊNCIAS BIBLOGRÁFICAS}

BARNI, V. Efeitos do Virus do estriado amarelo (GYSV) na produçăo de alho ov. Quitéria, sob diferentes condiçסes de cultiva Pelotas, 1992. 128 p. Dissertação (Mestrado em Produção Vegetal) Curso de PósGraduação em Agronomia, UFPEL, 1992.

COUTO, F.A.A. Efeito do espaçamento na produção de alho. Revista Ceres v. 10, n. 58, p. 288-289, 1958.

GARCIA, A., MORAES, E.C., MADAIL, J.C.M. et al. A cultura do alho. Pelotas: EMBRAPA/CNPFT, 1984. 76 p. (Circular Técnica, 8).

GASTAUD, C.S., CARINGI, L. de A.O. Ensaio de espaçamento na cultura do alho. In: REUNlão TÉCNICA ANUAL DE HORTALIÇAS 1983 \& 1984. Porto Alegre, RS, Ata.., Porto Alegre:SEAGRI-IPAGRO, 1985. $138 \mathrm{p}$. p. 412.

LOPES, M.C., BARBEDO, C.J. Influência de diferentes espaçamentos na cultura do alho $\mathrm{CV}$. Roxo Pérola de Caçador, em Bandeirantes-PR. Hort bras, v. 8, $\mathrm{n}$. 1, p. 49, 1990.

LUCINI, M.A., CHONAN, T. Área experimental com fileiras duplas Curi tibanos, SC, 1986. 5 p.(Mimeografado).

MASCARENHAS, M.H.T. Plantio $\theta$ espaçamento entre plantas na cultura do alho. Inf Agropec, v. 44, n. 48, p. 31-34, 1978.

MUELLER, S., BIASI, J. Efeito do espaçamento de plantio do alho, sobre o rendimento e seus componentes. In: CONGRESSO BRASILEIRO DE OLERICULTURA, 1982. Vitória, ES. Resumos... Vitória, ES. 1982. p. 252.

MUELLER, S., BIASI, J. Espaçamento de plantio do alho versus peso de bulbilhos. In: ENCONTRO DE HORTALIÇAS DA REGIāO SUL, 1990; ENCONTRO DE PLASTICULTURA DA REGlão SUL, 1990. Balneário Camboriú, SC. Resumos... Balneário Camboriú, SC. 1990. 127 p., p. 39.

OHM, H., SRIVASTAWA, R.P. Influence of the planting material and spacing on the growth yield of garlic. Indian J Hort, v. 34, n. 2, p. 152-156, 1977.

REGHIN, M.Y. Influência do espaçamento entre plantas na produção de alho vernalizado. Hort bras, v. $7, \mathrm{n}$. 1. p. 73, 1989.

SATURNINO, H.M., MASCARENHAS, M.H.T., FONTES, P. C.R. et al. Uso de diferentes arranjos de plantas em dois tipos de canteiros de alho (Allium sativum L). Revista de Olericultura v. 16, p. 41-44, 1976.

SOSA, P.M., LEANDRO, P.A. Efeito populacional na produção de alho semente da cultivar Lavinia. Hort bras, v. 4, n. 1, p. 45, 1986. 